\title{
Scanning Electron Microscopic Study of the Vascular Supply of the Equine Hoof
}

Tetsuo NASU, Takashi YAMANAKA ${ }^{1)}$, Masaaki NAKAI and Hiroyuki OGAWA ${ }^{2)}$

Departments of Veterinary Anatomy and ${ }^{1}$ Surgery, Faculty of Agriculture, Miyazaki University, Miyazaki 889-2192, and ${ }^{2}$ Animal

Hospital, Faculty of Agriculture, The University of Tokyo, Tokyo 113-8657, Japan

(Received 3 December 1997/Accepted 18 February 1998)

ABSTRACT. The blood supply in the equine hoof was studied by a microvascular casting corrosion technique and scanning electron microscopy in combination with observations of sections of the decalcificated digit. The dermal lamella was observed at the hoof wall and the dermal papilla at the other parts of the hoof. The microvascular architecture of the dermal lamella differed from that of dermal papilla. The vascular cast in the dermal papillar regions indicated that each papilla contained two central vessels (artery and vein), which ran parallel to each other, and the capillary plexus surrounding these vessels. In the dermal lamella region, the vessels consisted of thin parallel sheets arranged in vertical rows. Each sheet was made of branched arteries and veins, both of which were sandwiched between capillary plexus. At the distal and proximal parts of the wall, the vascular casts of the papillae merged to form the vascular sheets of the dermal lamella. - KEY wORDs: hoof, scanning electron microscopy, vascular supply.

J.Vet.Med.Sci.60(7): 855-858, 1998

The scanning electron microscope (SEM) has provided a more refined approach in the study of the vasculature system of organs. The microvascular corrosion casts of the digits have been examined with SEM in the horse $[4,5,8,9]$ and cattle [11]. These studies suggested the existence of arteriovenous anastomosis (AVA) in the dermal region of the hoof. However, the vascular structure of the transitional part between the dermal papilla and dermal lamella, a growing part of the hoof, is obscurely shown in previous reports. The purpose of this study is to investigate the blood vasculature system especially of the growing part of the horse hoof by using a SEM.

Six clinically healthy thoroughbred horses (1 mail and 5 females) were used in this study. After an intravenous injection of heparin $(100 \mathrm{unit} / \mathrm{kg})$ and xilagin $(1 \mathrm{mg} / \mathrm{kg})$ the horses were euthanasied with an intravenous injection of pentobarbitone sodium. The forelimbs were immediately amputated at the carpal joint. A polyethylene tube was inserted into the common digital artery of the forelimb and firmly ligated. The digital vessels were perfused with warm, normal saline solution containing 500 units of heparin until the perfusate became clear. The perfusate fluid was then replaced with $10 \%$ formalin. After perfusion, the coronary band, wall, sole and frog of the hoof were cut into blocks of $10 \times 10 \times 5 \mathrm{~mm}$ using a band saw. Each block was immersed separately in the same fixative. After decalcification by hydrochloric acid and formic acid, the specimens were dehydrated and embedded in paraplast. Sections were cut at $6 \mu \mathrm{m}$ and stained by hematoxylineosin and azan technique for light microscopic observation.

The perfusion of the limbs for vascular casts was carried out by the same method as for histological specimens. The casting medium (Mercox, Dainippon Ink, Co., Ltd.) was immediately injected through the same tube as that used for the perfusion of the limbs. After complete polymerization of the resin, the digits were frozen and cut by a band saw to get them into pieces. Each piece was immersed in toto in $1-3 \% \mathrm{NaOH}$ solution at $60^{\circ} \mathrm{C}$ for digestion of the soft tissues. Following maceration of the tissues, the specimen was washed gently with running water. The vascular cast specimen was air dried, coated with gold (E1030, Hitachi, Ltd., Tokyo), and examined with SEM (S-4100M, Hitachi, Ltd., Tokyo).

At the histological level the dermal papillae were observed in the coronary band, sole and frog. The dermis of the papilla in these parts was made up of the connective tissues that were supplied with small arteries, small veins and capillaries. A pair of artery and vein was observed at the central portion of each papilla surrounded by capillaries (Fig. 1). At the base of the papillae there was a vessel (AVA) communicating between the artery and the vein [1, $2,6]$. It had large endothelial cells protruding into the lumen (Fig. 2).

The dermal lamellae were observed at the hoof wall. They were deeply interdigitated with epidermis, and displayed numerous secondary dermal and epidermal

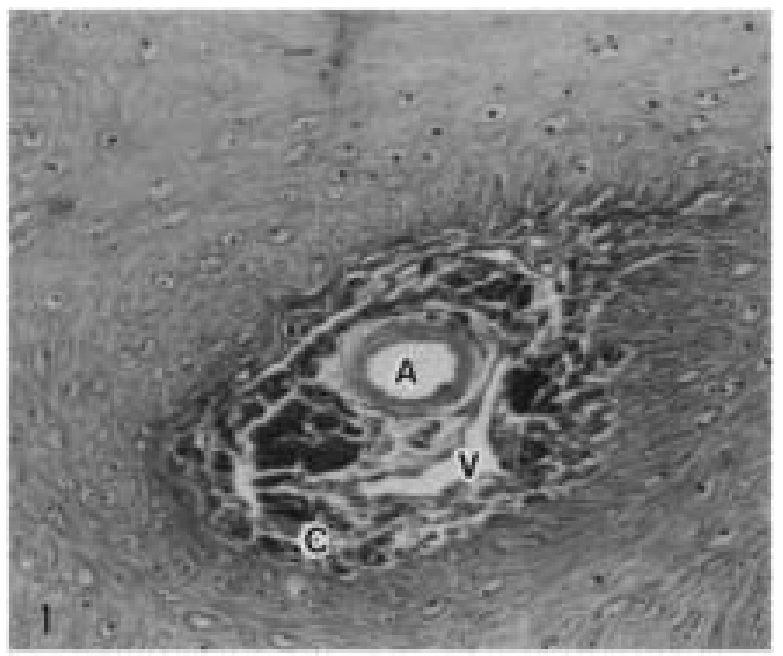

Fig. 1. Cross section of one dermal papilla. There are artery (A) and vein (V) in the papilla and the capillaries (C) around them. Azan staining. $\times 110$ 


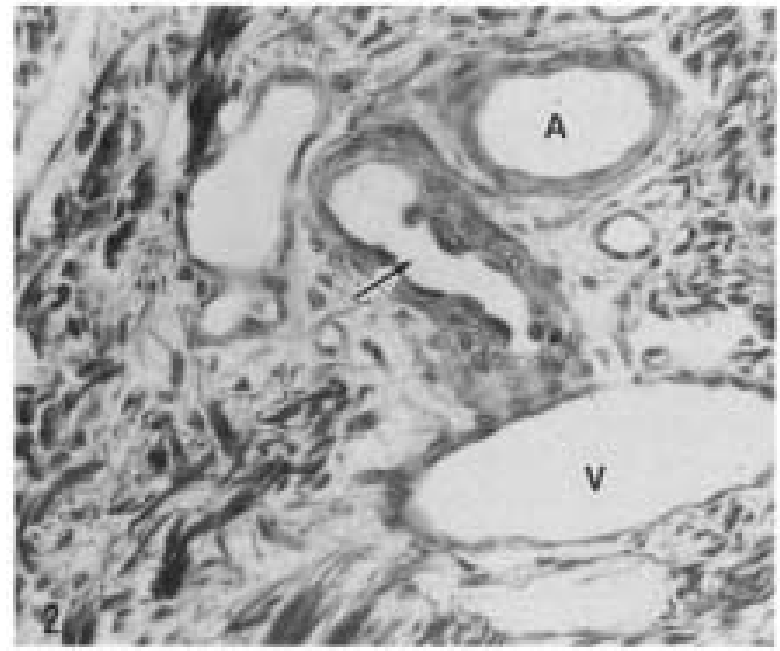

Fig. 2. The arteriovenous anastomose (AVA; arrow) between the artery (A) and vein (V). It was observed at the base of dermal papilla and had large endothelial cells protruding into the lumen. Azan staining. $\times 130$.

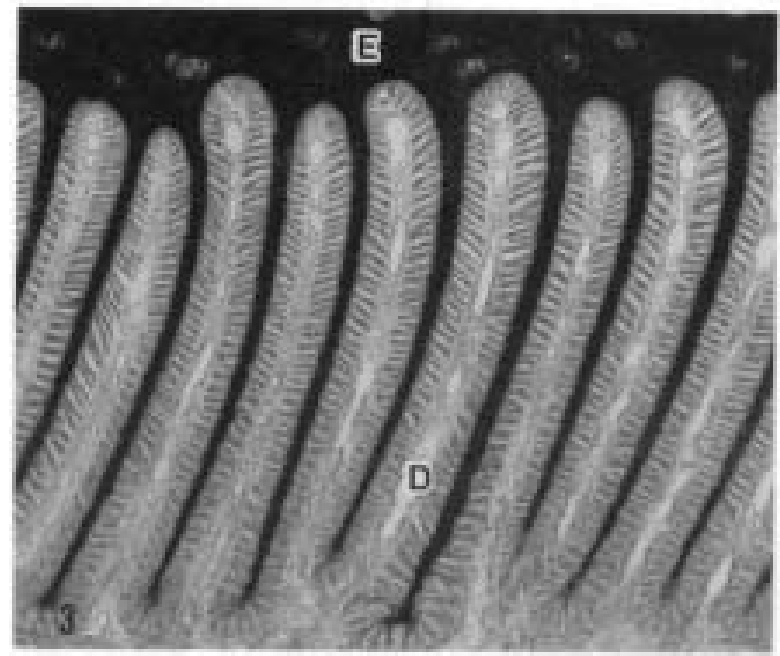

Fig. 3. The dermis (D) and epidermis (E) of the hoof wall interdigitating with each other. Their secondary interdigitation was observed. In the dermal lamella many vessels were observed. Azan staining. $\times 20$.

lamellae (Fig. 3). As in the case of the dermal papilla, the dermal lamella was made up of the connective tissues supplied with arteries, veins and capillaries. The veins and arteries coursed along the axis of the lamella and capillaries were observed on both sides of these vessels. A large calibre vein was located at the top of the lamella. At the basal part of the lamella, there were several AVAs with structures similar to those of the dermal papillae.

In the SEM observation the characteristic patterns of the vascular skeleton reflected the anatomy of the dermis. The vascular structure of the dermal papilla constituted the border that was made up of numerous tapering cone-shaped papillae (Fig. 4). Each papillar unit consisted of a pair of

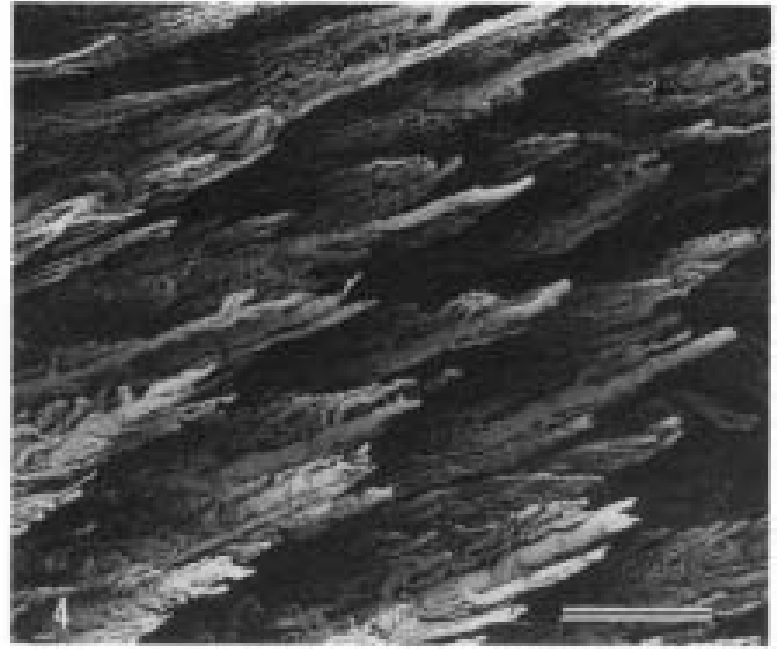

Fig. 4. The cast of the capillary plexus of the dermal papillae of the sole. There are many conical shaped papillae that fit into a socket in the epidermis of the coronary band. Bar $=540$ $\mu \mathrm{m}$.

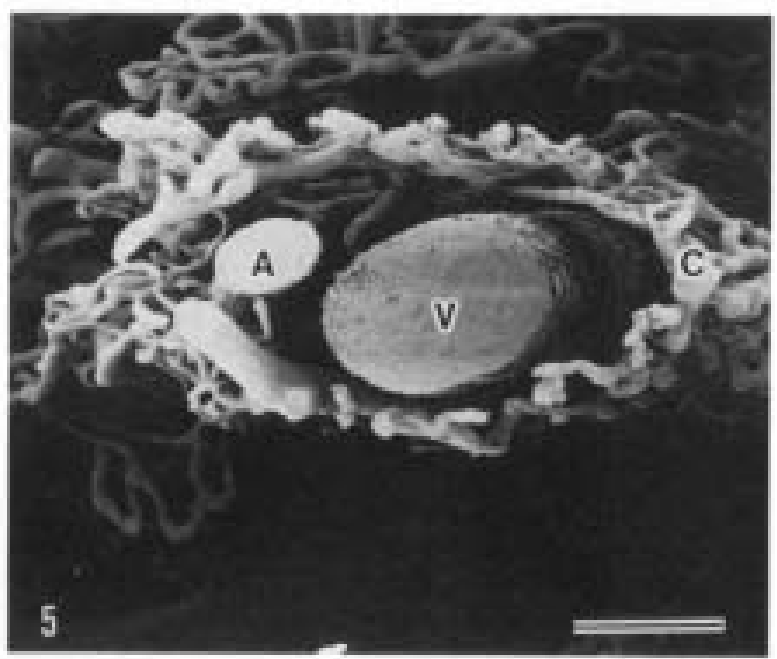

Fig. 5. Cut surface of one papilla of the sole dermis. Two central vessels (artery $[\mathrm{A}]$ and vein $[\mathrm{V}]$ ) are surrounded by the mesh of the capillary plexus (C). Bar $=120 \mu \mathrm{m}$.

central artery and vein surrounded by fine capillaries (Fig. 5). They were enmeshed in a sheath of cone and, in each papilla, the central artery coursed from the base to the top of the cone. The artery branched at intervals along the papilla to make the capillary plexus encircling the papillar unit, and each capillary drained into the central vein. At the apex of the cone, there was a vascular loop. From the observation of the cross section of one papilla, the artery was circular and small in calibre, whereas the vein was large in calibre (Fig. 5). The capillaries were the smallest in calibre and formed a meshed sheath. The papilla in the coronary band was longer than that in the other parts (sole and frog). At the base of the papillar unit, there was one spindle-shaped vessel (AVA) connecting the artery and vein 


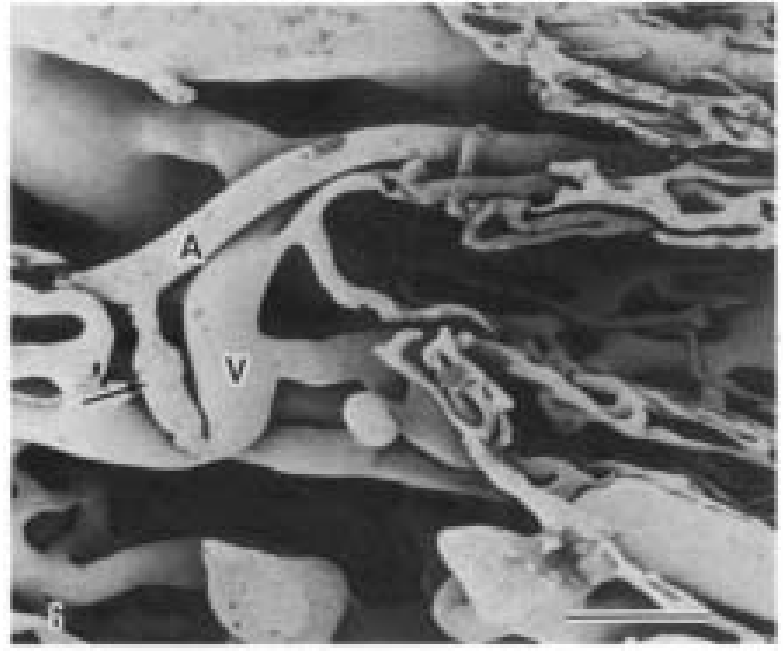

Fig. 6. The basal part of the dermal papilla in the sole. The spindle-shaped AVA (arrow) is observed between the artery (A) and vein (V) of the papilla. Bar=90 $\mu \mathrm{m}$.

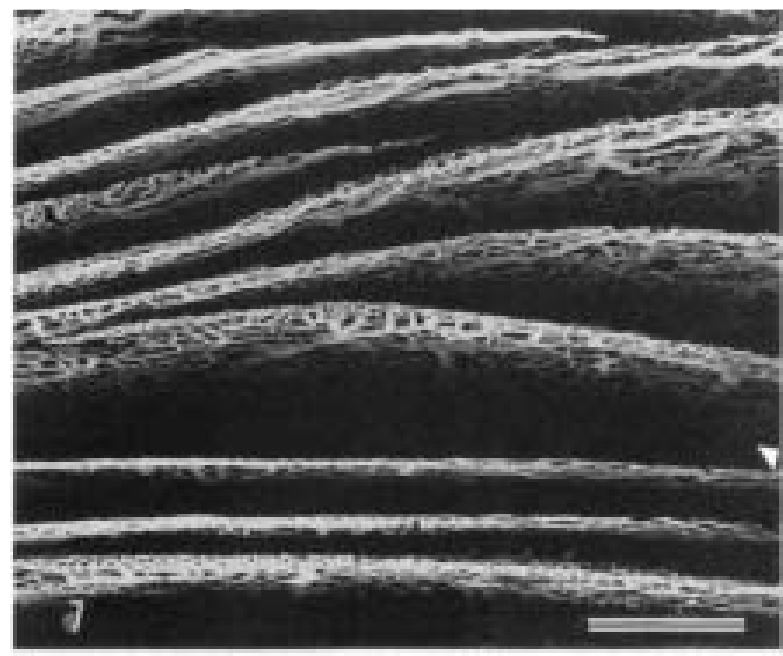

Fig. 7. The cast of the dermal lamellae of the wall. The thin parallel sheets are arranged in vertical rows. The spaces between the sheets correspond to the epidermal lamellae of the wall. Bar $=700 \mu \mathrm{m}$.

directly (Fig. 6).

In the lamellar region of the hoof dermis, the vascular cast made thin parallel sheets arranged in vertical rows. Each sheet was also made up of many vessels (Fig. 7). In the axial part of one lamella, the arteries were accompanied by axial veins and coursed to the surface of the hoof, where it branched to make the capillaries (Fig. 8). The capillaries extended throughout the dermal lamella to make a sheet of plexus. These capillary sheets were situated at the abaxial position of one lamella and drained into the vein that coursed axially to the peripheral part of the lamella to join large vein (marginal vein) [5] and then to the base of the lamella and finally into the efferent vein. The veins branched to make a large mesh.

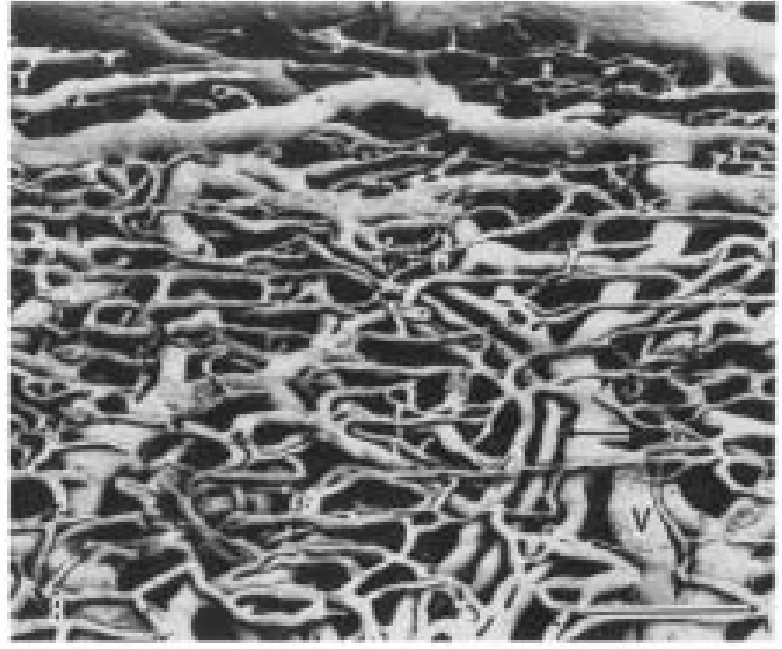

Fig. 8. Lateral view of the cast of the capillary plexus of the dermal lamella. The artery (arrow) and vein (V) course to the surface and anastomose with each other. These vessels are surrounded by the smaller calibre capillaries. Bar $=200 \mu \mathrm{m}$.

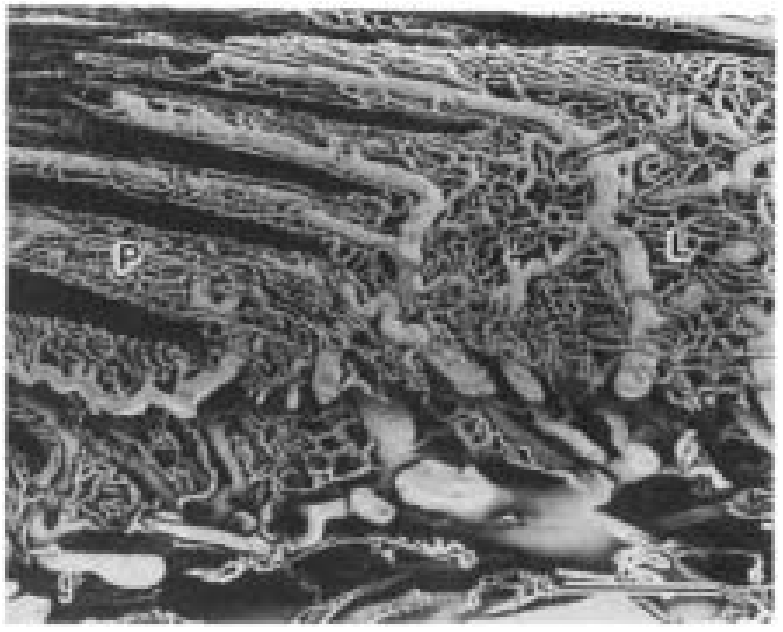

Fig. 9. Sagittal section of the toe. The papilla $(\mathrm{P})$ is observed at the distal end of the lamella (L) of the wall. The basal part of the papilla has merged with each other to form the dermal lamella. Bar $=600 \mu \mathrm{m}$.

The vascular structures of the proximal and distal ends of the wall transformed into those of dermal papillae. Near the wall, the papillae became flat and connected with each other. By the observation of the sagittal section of this part, a stack of papillae was formed and merged with the lamella (Fig. 9). A cross section of the merged part indicated that a pair of central vessels was surrounded by the capillary plexus mesh. The capillaries of the papilla surrounding the central artery and vein were connected to one another and formed one sheet of capillary plexus at the abaxial parts of the lamella. The central artery and vein of the papilla became the axial artery and vein of the lamella, respectively. 
The corrosion cast technique was served as a useful method of examining the vascular organization of the organs. This technique made it possible to get a complete filling of the most vessels of the digits. It was also possible to distinguish the vasculature of dermal papilla from that of dermal lamella with this technique. Though we could not distinguish the artery and vein by comparing their surface features of the resin cast as described elsewhere [3, 9, 11], the typical arterial cast of the horse showed a straight course, small diameter and was rounder in cross section when compared to the adjacent venous cast. The capillary was also small in calibre, but it showed several branches to form the capillary plexus. Though the $3 \mathrm{D}$ configuration of the vessels in the dermal papillae was basically the same as the previous reports $[9,11]$, the artery and vein ran parallel to one another and did not spiral around each other as reported by Pollitt and Molyneux [9]. In the dermal lamella, the basic vascular structure that the arteries and veins were sandwiched between the capillary plexus as was the same as that of the previous report [9], except that the artery and vein were not regularly arranged.

In the present histological observation, we could not observe so many AVAs in the dermal region as in the previous reports $[8,10]$. They were limited only at the basal portion of the papilla and lamella.

The dermal papilla is seen in the nail dermis of almost all mammals [7]. This is a basic structure of the nail which connects the dermis and epidermis. In the equine hoof, these structures were observed at the coronary band, frog and sole. In these parts, the dermis and epidermis do not suffer so much pressures and tensions. The dermal lamella is the characteristic structure in the ungulates, and it developes to the highest degree in the hoof of the solipeds. These animals show the striking modification of the dermis which are known as the lamella [7]. This structure makes a very firm connection between the epidermis and dermis. It is necessary for the nourishment of the thick epidermis and for resistance to the continuous pressure and tension between them during walking [7]. The presence of an extensive vascular network in the lamella might play a part in the shock absorbing mechanism of the hoof.

A new nail is formed in the matrix and it later protrudes outward [1]. Considering the vascular structures of the proximal and distal ends of the wall, we conclude that the dermal lamella is a particular feature of the dermal papilla in the growth of the hoof.

\section{REFERENCES}

1. Bloom, W. and Fawcett, D. W. 1975. A Textbook of Histology, 10th ed., B.W. Saunders, Philadelphia.

2. Iijima, T., Hasegawa, K. and Hirose, H. 1988. Cell Tissue Res. 252: 1-8.

3. Kardon, R. H. and Kessel, R. G. 1979. pp. 743-750. In: Scanning Electron Microscopy/1979/III (Becker, R.P. and Jahari, O. eds.), Scanning Electron Microscopy Inc., Chicago.

4. Marais, J. and Masty, L. 1988. Acta Anat. 133: 24-26.

5. Mishra, P. C. and Leach, D. H. 1983. Equine Vet. J. 15: 1421.

6. Molyneux, G. S., Haller, C. J., Mogg, K. and Pollit, C. C. 1994. Equine Vet. J. 26: 305-312.

7. Nickel, R., Schummer, A. and Seiferle, E. 1981. The Anatomy of the Domestic Animals, vol. 3. Verlag Paul Parey, Berlin.

8. Pollitt, C. C. 1993. Swiss Vet. 11: 72-74.

9. Pollitt, C. C. and Molyneux, G. S. 1990. Equine Vet. J. 22: 79-87.

10. Rooney, J. R. 1984. Equine Vet. Sci. 4: 182-183.

11. Vermunt,J.J. and Leach,D.H. 1992. N. Z. Vet. J. 40: 146-154. 